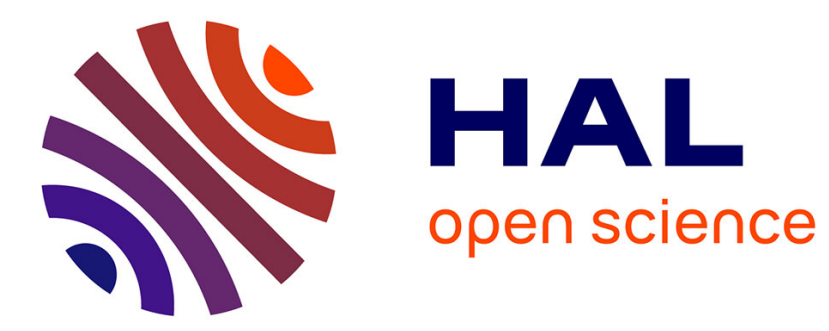

\title{
On the dynamic sliding with friction of a rigid block and of an infinite elastic slab
}

Michel Campillo, Ioan R. Ionescu, Jean-Claude Paumier, Yves Renard

\section{To cite this version:}

Michel Campillo, Ioan R. Ionescu, Jean-Claude Paumier, Yves Renard. On the dynamic sliding with friction of a rigid block and of an infinite elastic slab. Physics of the Earth and Planetary Interiors, 1996, 96 (1), pp.15-23. 10.1016/0031-9201(95)03113-8 . hal-00690527

\section{HAL Id: hal-00690527 \\ https://hal.science/hal-00690527}

Submitted on 26 Apr 2018

HAL is a multi-disciplinary open access archive for the deposit and dissemination of scientific research documents, whether they are published or not. The documents may come from teaching and research institutions in France or abroad, or from public or private research centers.
L'archive ouverte pluridisciplinaire HAL, est destinée au dépôt et à la diffusion de documents scientifiques de niveau recherche, publiés ou non, émanant des établissements d'enseignement et de recherche français ou étrangers, des laboratoires publics ou privés. 


\title{
On the dynamic sliding with friction of a rigid block and of an infinite elastic slab
}

\author{
Michel Campillo a, *, I.R. Ionescu ${ }^{\mathrm{b}}$, J.C. Paumier ${ }^{\mathrm{b}}$, Y. Renard ${ }^{\mathrm{b}}$ \\ abservatoire de Grenoble (UA CNRS 733), Université Joseph Fourier, Grenoble, France \\ ' Laboratoire Modelisation et Calcul, IMAG, Grenoble, France
}

We consider dynamic motions of two elastic systems undergoing frictional slip. The first one is the classical model of the frictional slider loaded through an elastic spring. The second one is an infinite elastic slab bounded by two planes which is in contract with a rigid body and submitted to shearing. Slip weakening and slip rate weakening friction laws are both considered. The two simple systems show very different qualitative behaviors. In the case of the slip dependent friction a slider moves with a single slip event when a critical stress level is reached. Under the same conditions, a series of slip events occur for the infinite slab. This difference between the behavior of the two systems is due to the important part played by inertia in the mass concentrated block slider model. In the case of slip rate weakening, the analysis of the problem for the infinite slab indicates a major difficulty: this problem has no unique solution if the rate of weakening exceeds a limit that is explicitly given. Whatever is the selection rule chosen to discriminate the solution, shocks will occur. The slip history obtained for the slab is very different from the one obtained with a block slider. For the infinite elastic slab the slip velocity exhibits sharp variations (shocks). On the contrary, a block slider does not exhibit this behavior. It is a clear example of the limitation of the use of such a simple analogy to describe the actual properties of the relative motions of two media in contact with friction.

\section{Introduction}

Since the moment release during earthquakes occurs essentially at depth, our knowledge of rupture process is limited by the poor resolution of the models deduced from seismological observations. Nevertheless the progress of inversion techniques makes it possible to reveal the main characteristics of the rupture such as duration or spatial heterogeneity (Hartzell and Heaton, 1983; Archuleta, 1984;

\footnotetext{
Corresponding author.
}

Beroza and Spudich, 1988; Cotton and Campillo, 1995). With these developments, there is a greater need for simple fault models which could account for the main features of fault behavior. Indeed, these last years these models were of crucial importance for the development of our understanding of the basic physical processes at work during an earthquake or a series of earthquakes. Two main classes of models played a central part in this discussion of how stresses accumulate, redistribute and dissipate during earthquakes. The first class of models follows the pioneering work of Burridge and Knopoff (1967). The elementary unit in this model consists of a 
totally concentrated mass sliding on a frictional surface and pulled by a spring. A series of such sliders linked by springs has shown a very rich and complex behavior similar by several ways to the one of observed seismicity (Burridge and Knopoff, 1967; Cao and Aki, 1986; Carlson and Langer, 1989). On the other hand, a single block slider has been used as an analog of a fault for the discussion of the effect of the non-linear friction laws deduced from laboratory experiments ( $\mathrm{Li}, 1987$; Scholz, 1990).

Indeed, the same problem of non-linear friction can be adressed from the point of view of a model based on the equation of elasticity, i.e. the contact problem at the boundary of an elastic body. Such crack models form a second class of models and were studied numerically in the dynamic 2D case (Andrews, 1985; Okubo, 1989; Harris and Day, 1993; Cochard and Madariaga, 1994) as well as in the quasi-static 3D case (Rice, 1993). We intend here to compare these two approaches in the simplest geometrical cases: a single block and an infinite elastic slab sliding on a frictional surface. Our aim is to understand the importance of the friction law for the 1D problem and to identify the theoretical problems associated with non-linearity. Specifically, we shall examine the existence and unicity of the solutions. Our discussion will be limited to the case of simple extreme cases of slip weakening and slip rate weakening behaviors.

\section{Description of the models}

Let us consider $\Omega$ a body which is in contact with a rigid foundation along a contact surface $\Gamma_{f}$. We denote by $\sigma$ the stress tensor, $u$ the displacement, $\dot{u}$ the velocity, $n$ the unit vector outward on $\Omega$ and normal to $\Gamma_{f}, \sigma_{n}=\sigma n \cdot n$ the normal stress, $\sigma_{\tau}=\sigma n$ $-\sigma_{n} n$ the tangential stress, $\dot{u}_{\tau}=\dot{u}-(\dot{u} \cdot n) n$ the tangential velocity (slip rate) and $\mu \geq 0$ the friction coefficient.

The contact interface condition on $\Gamma_{f}$ reads:

$u \cdot n \leq 0, \quad(u \cdot n) \sigma n=0$,

$\left|\sigma_{\tau}\right| \leq \mu\left|\sigma_{n}\right|, \quad\left(\left|\sigma_{\tau}\right|-\mu\left|\sigma_{n}\right|\right)\left|\dot{u}_{\tau}\right|=0$.

there exists $\quad \gamma \geq 0$ such that $\dot{u}_{\tau}=-\gamma \sigma_{\tau}$.

In our systems we deal with the special case where $\sigma_{\tau}, \sigma_{n}, u_{\tau}, u_{n}, \dot{u}_{\tau}$ and $\dot{u}_{n}$ are scalar and, for simplicity, we have $\sigma_{n}<0, \dot{u}_{\tau} \geq 0, u_{n}=0$ and $u_{\tau} \geq$ 0.

In the first system we consider a rigid block called the slider which is in contact with friction on a rigid foundation and which is submitted to a traction force by means of a spring pulled at velocity $V$ (see Fig. 1). Consequently the mass is concentrated on the contact surface and from the balance law we get the equations

$$
\begin{aligned}
& m \ddot{u}(t)-G\left(\dot{D}_{0}+V t-u(t)\right) \\
& \quad=-\mu S, \quad \text { if } \quad \dot{u}(t)>0 \\
& G\left|D_{0}+V t-u(t)\right| \leq \mu S, \quad \text { if } \quad \dot{u}(t)=0 \\
& \dot{u}(0)=0, \quad u(0)=u_{0} .
\end{aligned}
$$

where $m$ is the mass of the slider, $-S<0$ is a prescribed normal force applied on the top of the slider, $u_{0}$ is the initial position and $G\left(D_{0}-u_{0}\right)$ is the initial tension of the spring whose elastic modulus is $G$.

In the second system we consider the one dimensional shearing of an infinite linear elastic slab (with elastic coefficients $\lambda$ and $G$ ) bounded by the planes $x=0$ and $x=h$ (as in Fig. 1). On the plane $x=0$ the slab is in contact with friction with a rigid

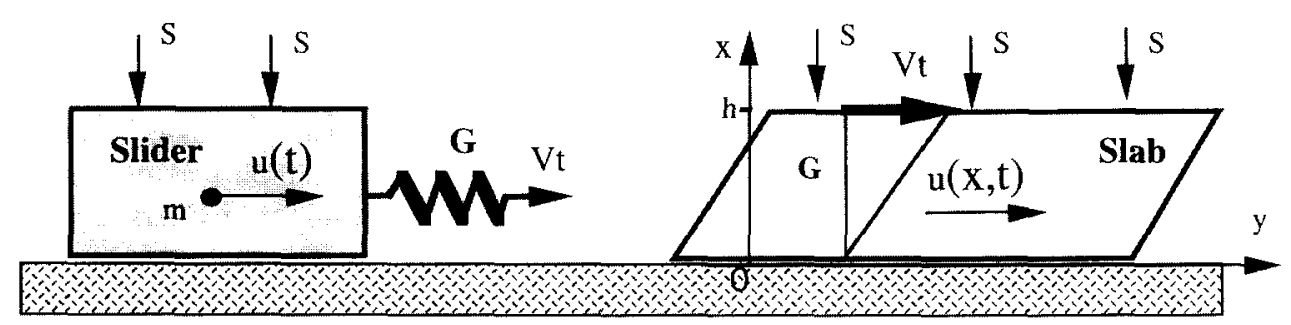

Fig. 1. Description of the two systems, the slider and the slab, in contact with friction on a rigid foundation. 
foundation. At $x=h$ the slab is dragged with a tangential velocity $V$ from an initial position $D_{0}$ and it is pushed with a uniform normal stress $-S$. We assume that the displacement field vanishes in the $z$-direction (perpendicular to the $x$ and $y$ axes) and has the value $-S x /(\lambda+2 G)$ with respect to the $x$-direction, following Hooke's law. We denote by $u$ the horizontal displacement (with respect to the $y$-direction) and we suppose that it depends on $t$ and $x$, i.e. $u=u(x, t)$. In this way we get on the frictional boundary $x=0$ the normal and tangential stress $\sigma_{n}=-S, \quad \sigma_{\tau}=-G \partial_{u}(0, t) / \partial_{x}$. Assuming that $\dot{u}(0, t) \geq 0$, from (1)-(3) and the equations of the elastodynamic, we get the following initial and boundary value problem:

$\rho \ddot{u}(x, t)=G \frac{\partial^{2} u}{\partial x^{2}}(x, t)$,

$G \frac{\partial u}{\partial x}(0, t)=\mu S, \quad$ if $\quad \dot{u}(0, t)>0$,

$G\left|\frac{\partial u}{\partial x}(0, t)\right| \leq \mu S, \quad$ if $\quad \dot{u}(0, t)=0$,

$u(h, t)=D_{0}+V t$

$\dot{u}(x, 0)=V \frac{x}{h}, \quad u(x, 0)=u_{0}+\left(D_{0}-u_{0}\right) \frac{x}{h}$.

where $\rho$ is the density and $u_{0}$ is the initial displacement (slip) at $x=0$.

For each of these two systems we consider two cases:

Slip weakening case. The coefficient $\mu$ depends on the slip displacement (see Fig. 2):

$\mu=\mu(u(t))$ for the slider:

$\mu=\mu(u(0, t))$ for the slab.
Slip rate weakening case. The coefficient $\mu$ depends on the slip rate $\dot{u}(0, t)$ (se Fig. 2):

$\mu=\mu(\dot{u}(t))$ for the slider:

$\mu=\mu(\dot{u}(0, t))$ for the slab.

\section{Mathematical aspects}

For the first system (slider) no mathematical special difficulties appear in the analysis of ordinary differential Eq. (4)-(6) in the slip or slip rate weakening cases.

For the second system (slab) the partial differential equations are hyperbolic and can be reduced (for details see Ionescu and Paumier, 1993, 1994) to the following equation on the friction boundary:

$$
\sqrt{\rho G} \dot{u}(0, t)+\mu S=\beta(t), \quad \text { if } \quad \dot{u}(0, t)>0,
$$

$$
|\beta(t)| \leq \mu S, \quad \text { if } \quad \dot{u}(0, t)=0 \text {, }
$$

where, for a time $t \in\left[0, T^{*}\right]$ with $T^{*}=h \sqrt{\rho / G}$, the function $\beta$ is given by: $\beta(t)=G\left(V t+D_{0}-\right.$ $\left.u_{0}\right) / h$. For $t>T^{*}$ it is possible to obtain an analytical expression for the function $\beta$ which has to be modified in order to take into account the reflected wave and the boundary condition at $x=h$.

In the first case, where $\mu=\mu(u(0, t))$, this equation is an ordinary differential equation for $u(0, t)$ and the solution exists and is unique. Consequently, the slab problem with slip dependent friction is mathematically well-posed.

In the second case, where $\mu=\mu(\dot{u}(0, t))$, this equation is a scalar equation for the slip rate $\dot{u}(0, t)$.
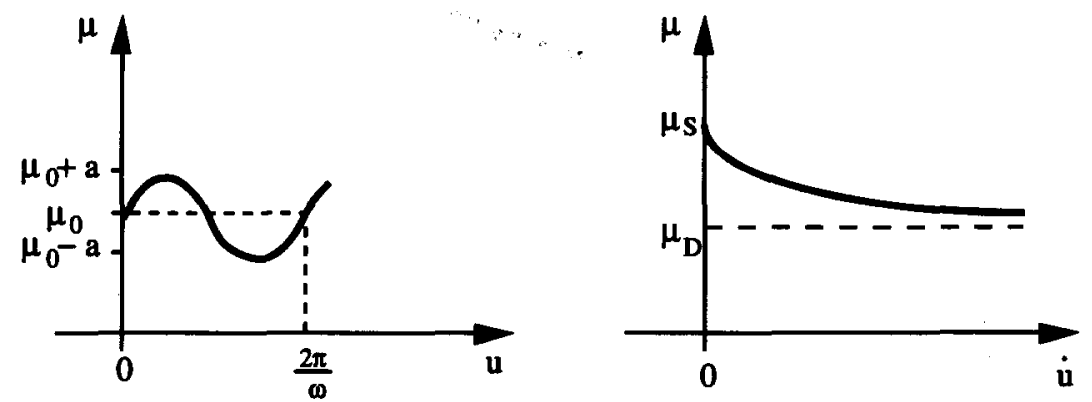

Fig. 2. In the first case the coefficient of friction $\mu$ depends on the displacement and in the second case it depends on the slip velocity. 

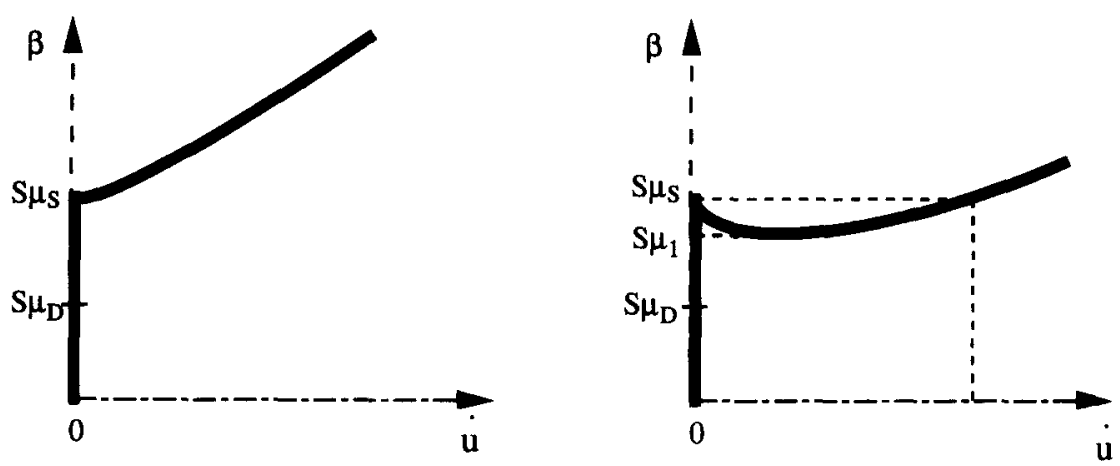

Fig. 3. Illustration of the regular and irregular regimes of the system depending upon the characteristics of the slip rate dependance. The solutions $(\dot{u}, \beta)$ belong $K$ that is plotted in thick solid line in the case of regular behavior (left side) and in the case of irregular behavior (right side).

If we denote by $g(s)=\sqrt{\rho G} s+\mu(s) S$ then (12)(13) may be written as

$$
\begin{aligned}
& (\dot{u}(0, t), \beta(t)) \in K=\{(s, y): \quad y=g(s) \text { or } \\
& \quad s=0,|y|<g(0)\},
\end{aligned}
$$

where the set $\boldsymbol{K}$ is plotted in the Fig. 3. Two qualitative behaviors are possible depending on the shape of $\mu$. In the first one, called the regular behavior, $g$ is increasing and there is a unique solution $\dot{u}(0, t)$ for all $\beta(t)$. In the second one, called the irregular behavior, $g$ is not anymore in- creasing. The solution $\dot{u}(0, t)$ is unique if $\beta(t)<S \mu_{1}$ or $S \mu_{s}<\beta(t)$, but there are three solutions for $S \mu_{1}$ $<\beta(t)<S \mu_{s}$.

However, since the solution of the problem is not uniquely determined for the irregular behavior, we need a criterion to select between the three solutions the one which is more appropriate to a physical interpretation. Whatever is the selection rule chosen to discriminate the solution, shocks will occur. A possible choice for this criterion is the (perfect) delay convention of the catastrophe theory (see for

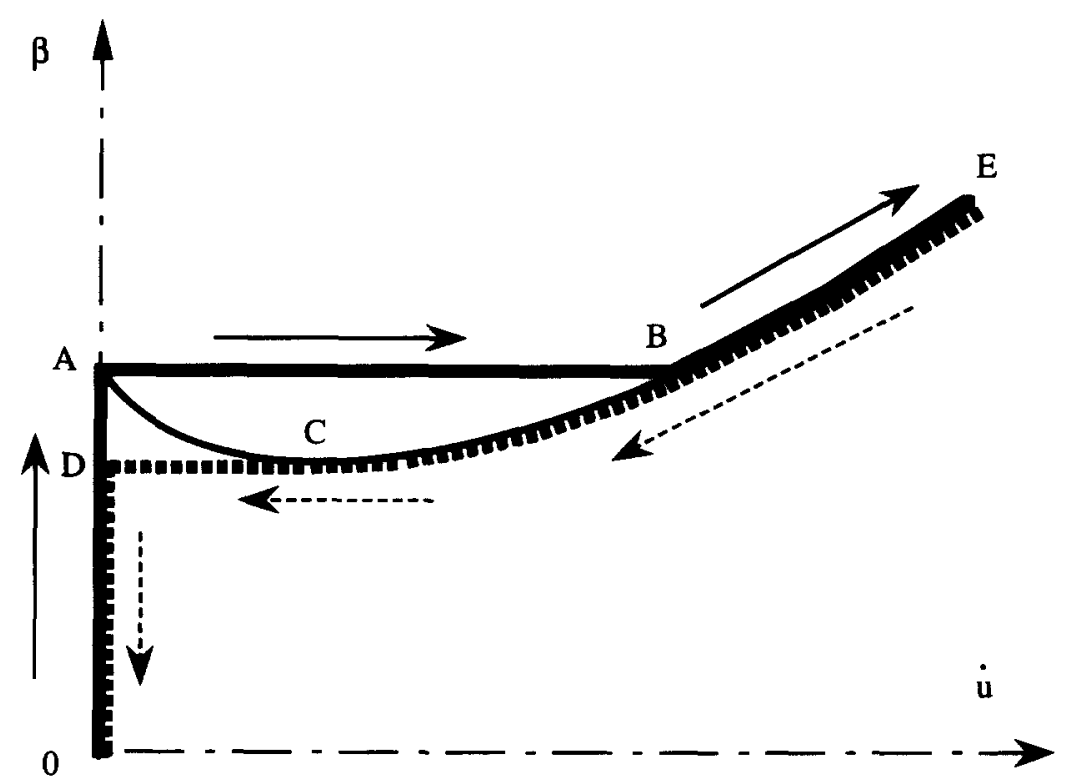

Fig. 4. Using the delay convention of catastrophe theory to select a unique solution. 
instance Poston and Stewart, 1978): the system only jumps when it has no other choice. In this way (see Fig. 4) different paths of solutions are obtained in acceleration (path ODABE, solid arrows) and deceleration (path EBCDO, dotted arrows) processes and a hysteresis phenomenon occurs. As it follows from Ionescu and Paumier (1996) the perfect delay convention is not related to a simple energy criterion.

One may notice that the delay criterion, which we give explicitely here, is implicitly present in the analysis of many physical problems. For instance, even in the case of the slider, it is implicitly accepted that the slider will keep motionless as long as possible when the load is increasing.

\section{Comparison between the systems}

The aim of this paragraph is to point out the difference which appear in the numerical simulations of a very slow loading process for the two systems, considered above, with the two friction law types. Since we are interested in a qualitative comparison, the physical constants have not a great importance. However we tried to choose them as 'realistic' as possible. Since we deal with very simple models, which are rather far from the physical reality, it is not always possible to give physical interpretation of our choice. Having in mind that for both systems we have a very slow loading process, $V=0.05 \mathrm{~m} \mathrm{year}^{-1}$, we have chosen the initial data close to an unstable position.

For the slab system we have considered $\rho=2800$ $\mathrm{kg} \mathrm{m}^{-3}, h=10^{4} \mathrm{~m}, c=\sqrt{G / \rho}=3500 \mathrm{~m} \mathrm{~s}^{-1}, G=$ $G_{\text {slab }}=c^{2} \rho$ and $S=10^{8} \mathrm{~N} \mathrm{~m}^{-2}$. In the slip weakening case we have chosen $u_{0}=u_{c}+10^{-3} \mathrm{~m}$ where $u_{c}$ is such that $d \mu\left(u_{c}\right) / d u=0$ and $D_{0}$ such that the initial shear stress $G\left(D_{0}-u_{0}\right) / h$ is equal to $\mu\left(u_{c}\right) S$. In the slip rate weakening case we have $u_{0}=0$ and $D_{0}$ such that initial shear stress $G D_{0} / h$ is equal to $\mu(0) S$.

For the block slider system we consider $m=\rho$.
RIGID BLOCK
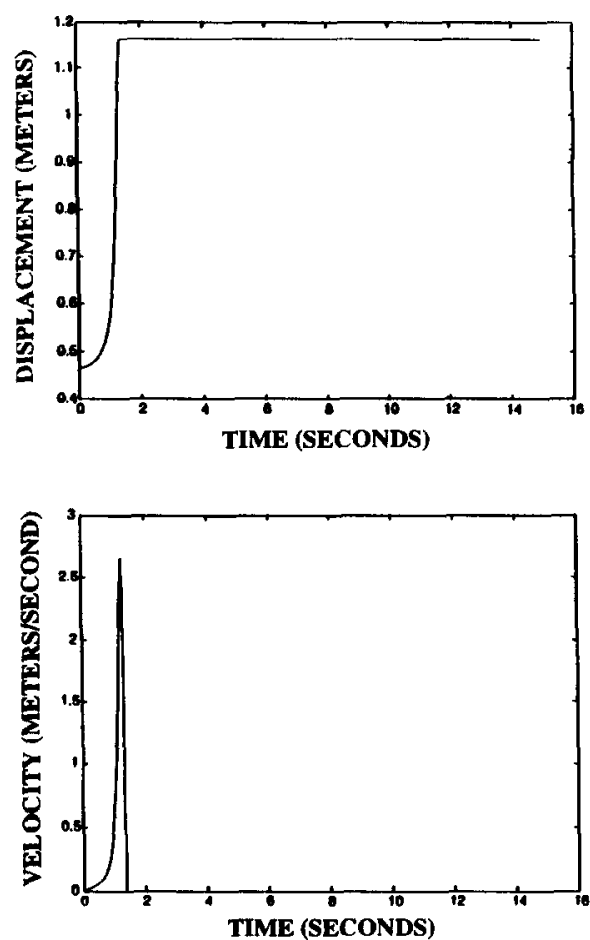

ELASTIC SLAB
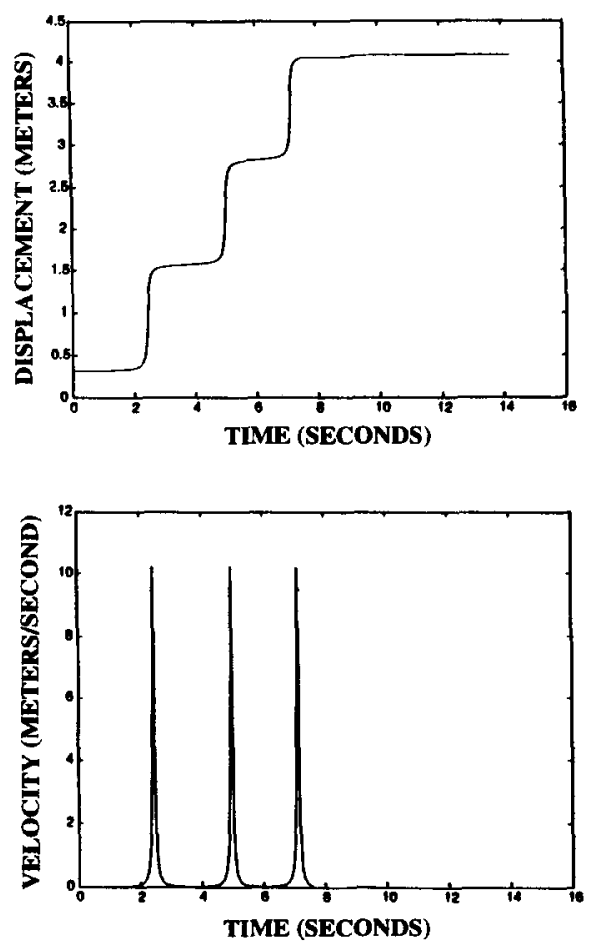

Fig. 5. Comparison between the systems in the case of slip dependent friction. 
$10^{9} \mathrm{~m}^{3}, G=G_{\text {slab }} \cdot 10^{4} \mathrm{~m}$ and $S=10^{14} \mathrm{~N}$. In the slip weakening case we have chosen $u_{0}$ such that $S d \mu\left(u_{0}\right) d u+G=0$ and $D_{0}=S \mu\left(u_{0}\right) / G+u_{0}+$ $10^{-3} \mathrm{~m}$. In the slip rate weakening case $u_{0}=0$ and $D_{0}=S \mu(0) / G$.

\subsection{The case of slip dependent friction}

The importance of slip dependent friction was demonstrated from theoretical and experimental points of view (Brace and Byerlee, 1966; Byerlee, 1967, 1970; Byerlee and Brace, 1968). In our study we do not intend to discuss the details of a particular friction law but rather we want to investigate the qualitative implications of a slip dependent friction. For this reason, we choose to consider here a friction law given by the following dependence of stress with displacement:

$\mu=\mu_{0}+a \sin (\omega u)$.

where $\omega=5 \mathrm{~m}^{-1}, \mu_{0}=1$ and $a=0.5$ This friction law is illustrated in Fig. 2. It corresponds to a process of slip weakening followed by slip hardening after a certain displacement. It is similar to an example discussed in Scholz (1990). Fig. 5 shows the slip, the slip velocity and tangential stress obtained for a block slider. As noticed by Scholz (1990), the block moves when a critical strain level is reached, thus releases significantly the stress level and stops. Then it will remain immobile for the long period needed to reach again the critical stress level. This very simple behavior suggests that with a slip dependent friction one cannot expect to observe stick slip events. Considering now the case of the infinite slab with the same friction law we obtained the results shown in Fig. 5.

A striking qualitative difference of behavior between the two models appears with the existence of several pulses in the case of the slab. It shows that in this case the stress release occurs with a series of stick-slip-like events on a very short time scale. The stress history on the frictional surface is governed by
RIGID BLOCK
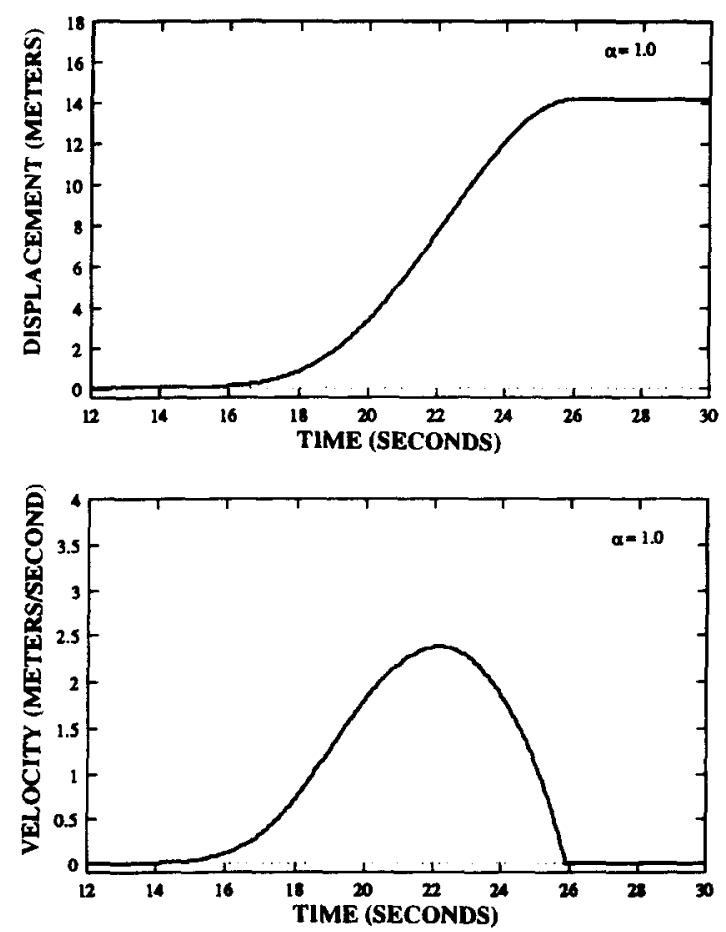

ELASTIC SLAB
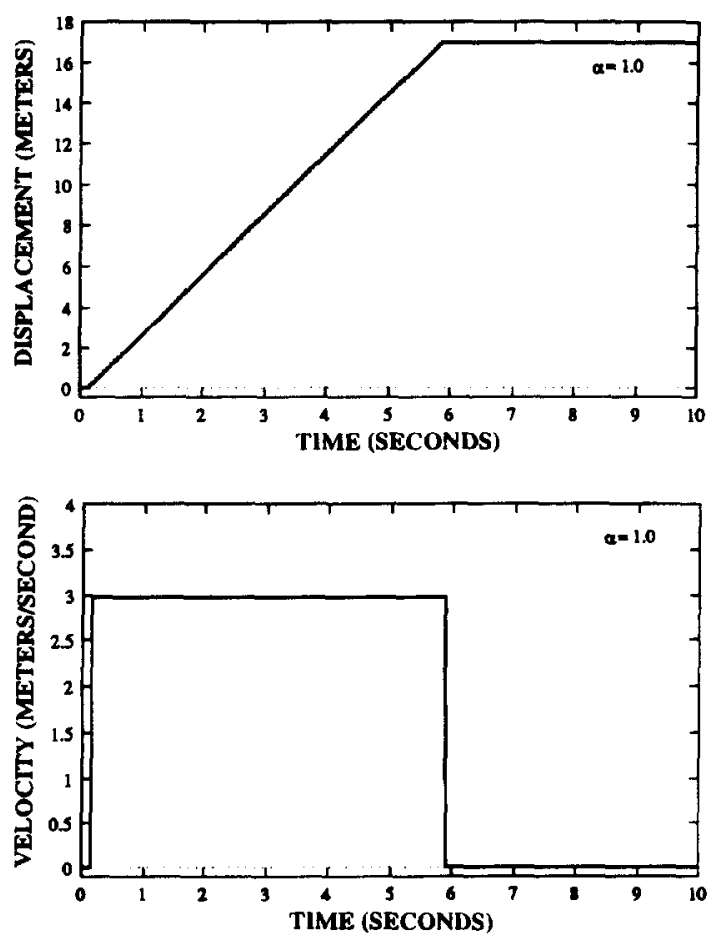

Fig. 6. Comparison between the systems in the case of slip rate dependent friction when $\alpha=1$ (irregular regime). 
two different phenomena. The first one is related with the friction law and results in the occurrence of several slip pulses which produce waves that propagates in the slab. The second one is related to the reflection of the primary waves on the boundary of the slab. The arrival of the reflected waves at the frictional surface produces an important stress release responsible for the complete arrest of the slip. Therefore, the number of slip events depends only on the thickness of the slab and on the wave velocity in this very simple model. This result from the 1D model could be important for the understanding of seismological records since it could indicate the possibility of several slip velocity pulses during a sliding event if such a property is retained in $3 \mathrm{D}$.

\subsection{The case of slip rate dependence}

It is widely accepted that slip rate weakening plays an important part for the behavior of actual faults (see for instance Scholz, 1990). We consider here the following dependence:

$\mu=\mu_{D}+\frac{\mu_{S}-\mu_{D}}{1+\alpha \dot{u}}$,

where $\mu D=0.3, \mu_{s}=0.8$ and $\alpha>0$ is a parameter that governs the shape of $\mu$. This dependance is shown in Fig. 2. We first consider $\alpha=1 \mathrm{~s} \mathrm{~nm}^{-1}$. In the case of the slider, as it is shown in Fig. 6 the slip rate is a continuous function of time. On the contrary, for the slab, the slip rate is characterized by sudden jumps. Again, the two systems present very different qualitative behaviors.

To further investigate the discontinuous behavior of the slab, we choose $\alpha$ to be very close to the limit between the regular and irregular regimes which we defined previously. This limit, denoted by $\alpha_{c}$, is obtained from $d g(0) / d s=0$. Therefore $\alpha_{c}$ is equal to $\sqrt{\rho G} / S\left(\mu_{S}-\mu_{D}\right)$ which is $0.196 \mathrm{~s} \mathrm{~m}^{-1}$ in our case. In the computations presented in Fig. 7, we
RIGID BLOCK
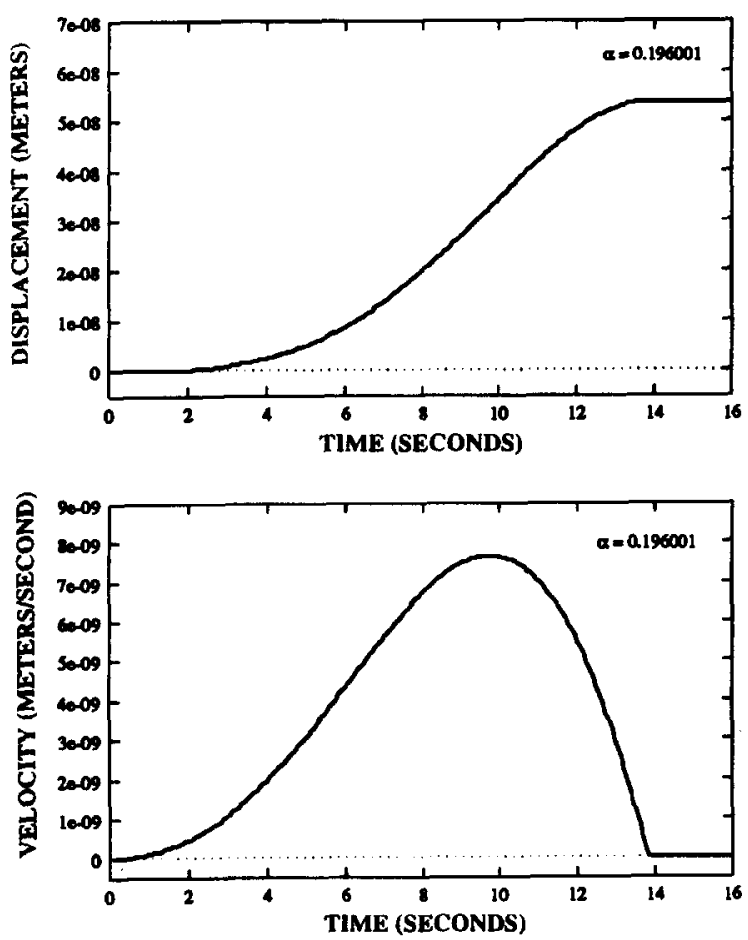

BLASTIC SLAB
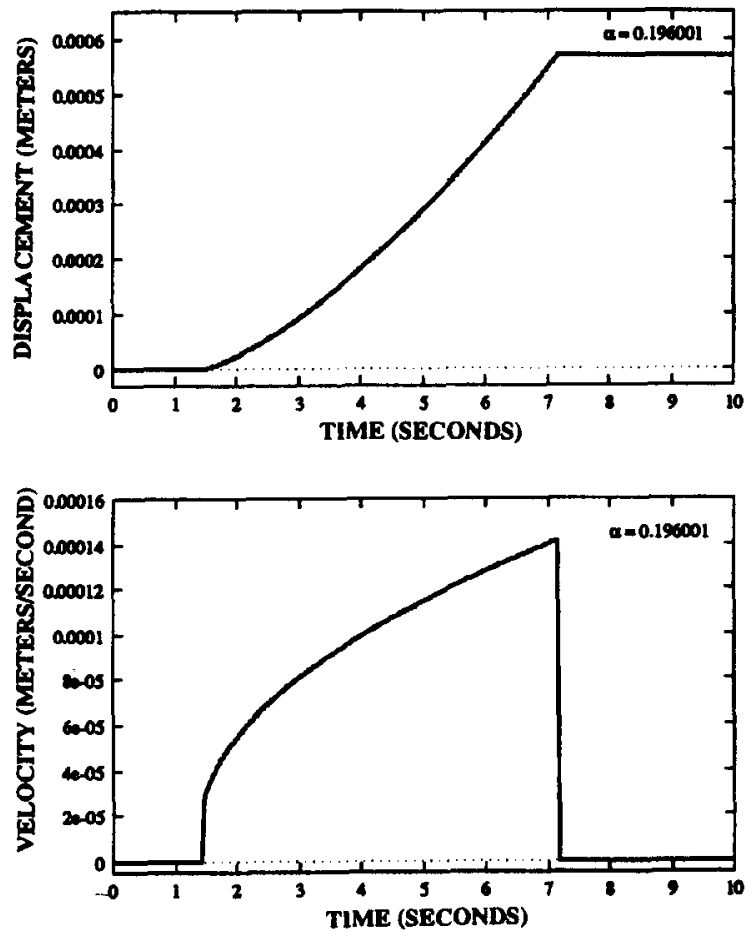

Fig. 7. Comparison between the systems in the case of slip rate dependent friction when $\alpha$ is chosen very close to the limit $\alpha_{c}$ between the regular and irregular regime. 
choose $\alpha=\alpha_{c}+10^{-6} \mathrm{~s} \mathrm{~m}^{-1}$. While the slip rate is very small in this case, we note the presence of two shocks at the begining and the end of the event. The first shock is directly produced by the friction law (shock $\mathrm{AB}$ in Fig. 4) while the second shock also involves the reflected stress wave that produced a discontinuity in $\beta(t)$. This indicates that, in the whole irregular regime of the slab, the slip rate is dominated by shocks while the slider model is characterized by smooth slip rate.

We remark that when $\alpha$ is larger enough (for instance $\alpha>3 \alpha_{c}$ ) the system behaves as for the classical Coulomb's law $\mu(0)=\mu_{S}, \mu(s)=\mu_{D}$ for $s>0$, where $\mu_{S}$ and $\mu_{D}$ are the static and dynamic friction coefficients.

\section{Conclusion}

The two simple systems under consideration show very different qualitative behaviors. This observation holds for both slip and slip rate dependent friction. In the case of the slip dependent friction considered here, a slider moves with a single slip event when a critical stress level is reached. Under the same conditions, a series of slip events occur for the infinite slab. This difference between the behavior of the two systems is due to the important part played by inertia in the mass concentrated block slider model. In the case of slip rate weakening, the analysis of the problem for the infinite slab indicates a major difficulty: this problem may have not a unique solution. Whatever is the selection rule chosen to discriminate the solution, shocks will occur. This point indicates the need to introduce in the model an extra-condition deduced from physical considerations. One may be afraid that, in numerical solutions, this condition is implicitly present in the numerical scheme independently of the assumed physical hypothesis. We choose here the solution corresponding to the perfect delay convention. With this assumption, the slip history obtained for the slab is very different from the one obtained with a block slider. For the infinite elastic slab the slip velocity exhibits sharp variations (shocks) in a wide range of the parameter $\alpha$ which governs the shape of the friction law.

\section{Acknowledgments}

We thank Alain Cochard for his careful review of the paper.

\section{References}

Andrews, D., 1985. Dynamic plane strain shear rupture with a slip-weakening friction law calculated by a boundary integral method. Bull. Seismol. Soc. Am., 75: 1-21.

Archuleta, R.J., 1984. A faulting model for the 1979 Imperial valley earthquake, J. Geophys. Res., 89: 4559-4585.

Beroza, G.C. and Spudich, P., 1988. Linearized inversion for fault rupture behavior: Application to the 1984 Morgan Hill. California, earthquake, J. Geophys. Res., 93: 6275-6296.

Brace, W.F., Byerlee, J.D., 1966. Stick-slip as a mechanism for earthquakes. Science, 153: 990-992.

Burridge, R. and Knopoff, L., 1967. Model and theoretical seismicity. Bull. Seismol. Soc. Am., 57: 341-371.

Byerlee, J.D., 1967. Frictional characteristics of granite under high confining pressure. J. Geophys. Res. 72, (14): 3639-3648

Byerlee, J.D., 1970. The mechanism of stick-slip. Tectonophysics. 9: $475 \sim 486$.

Byerlee, J.D. and Brace, W.F., 1968. Stick slip, stable sliding and earthquakes - Effect of rock type, pressure, strain rate and stiffness. J. Geophys. Res., 73: (18): 6031-6037.

Cao, T. and Aki, K., 1986. Seismicity simulation with a rate- and state-dependent friction. Pure Appl. Geophys, 124: 487-513.

Carlson, J.M. and Langer, J.S., 1989. Mechanical model of an earthquake fault. Phys. Rev. A, 40: 6470-6484.

Cochard, A. and Madariaga, R., 1994. Dynamic faulting under rate dependent friction. Pure Appl. Geophys., 142: 419-445.

Cotton, F. and Campillo, M., 1995. Frequency domain inversion of strong motions: Application to the 1992 Landers earthquake. J. Geophys. Res., 100: 3961-3975.

Harris, R.A. and Day, S.M., 1993. Dynamics of fault interaction parallel strike-slip faults. J. Geophys. Res., 98: 4461-4472.

Hartzell, S.H. and Heaton, T.H., 1983. Inversion of strong ground motion and teleseismic waveform data for the fault rupture history of the 1979. Imperial Valley, California, earthquake Bull. Seismol. Soc. Am., 73: 1553-1583.

Ionescu, I.R. and Paumier, J.-C., 1993. Dynamic stick-slip motions with sliding velocity - dependent friction. Comptes Rendus del'Acad. Sci. Paris. 316(s.I): 121-125.

Ionescu, I.R. and Paumier, J.-C., 1994. On the contact, problem with slip rate dependent friction in elastodynamics, Eur. $\mathrm{J}$ Mech., A/Solids. 13(4): 555-568.

Ionescu, I.R. and Paumier, J.-C., 1996. On the contact problem with slip displacement friction in elastostatics. Int. J. Eng. Sci., in press.

Li. V.C., 1987. Mechanics of shear rupture applied to earthquake zones. In: B.K. Atkinson (Editor). Fracture Mechanics of Rocks. Academic Press. 
Okubo, P., 1989. Dynamic rupture modeling with laboratory-derived constitutive relations. J. Geophys. Res., 94: 1232112335.

Poston, T. and Stewart, I., 1978. Catastrophe Theory and its Applications. Pitman.
Rice, J.R., 1993. Spatio-temporal complexity of slip on a fault. J. Geophys. Res., 98: 9885-9907.

Scholz, C., 1990. The Mechanics of Earthquakes and Faulting. Cambridge University Press. 